\title{
Differential expression of Dickkopf-1 among non-small cell lung cancer cells
}

\author{
XIAO JUN XIANG ${ }^{1}$, YA WEN LIU ${ }^{2}$, DIAN DIAN CHEN ${ }^{1}$ and SHUANG YU ${ }^{1}$ \\ ${ }^{1}$ Department of Oncology, The First Affiliated Hospital of Nanchang University; \\ ${ }^{2}$ Department of Oncology, Maternal and Child Health Hospital, Nanchang, Jiangxi 330006, P.R. China
}

Received March 15, 2014; Accepted December 12, 2014

DOI: $10.3892 / \mathrm{mmr} .2015 .3654$

\begin{abstract}
Dickkopf-1 (DKK1) is a negative regulator of the Wnt/ $\beta$-catenin signaling pathway, which is expressed in various human cancers. It was hypothesized that DKK1 was oncogenic and involved in invasive growth in non-small cell lung cancer (NSCLC) cells. The present study aimed to investigate whether DKK1 gene expression levels differ among various NSCLC cells. The DKK1 expression pattern was analyzed in various human NSCLC cell lines and tissues. The DKK1 protein and gene expression levels were quantified using immunoblotting, polymerase chain reaction analysis and immunohistochemistry. The majority of the lung cancer cell lines analyzed revealed increased expression levels of DKK1. Furthermore, DKK1 expression was highly transactivated in the majority of these cancer cell lines. Clinical samples were obtained from 98 NSCLC patients for immunohistochemical analysis. Of the 98 samples analyzed, 62 (63.3\%) demonstrated positive staining for DKK1, whereas the remaining 36 (37\%) exhibited negative staining. However, no immunohistopathological staining was detected in normal tissues. The relative effects of DKK1 were assessed in a high-expression cell line (LTEP-a-2) and a low-expression cell line (95D). The differential expression of genes involved in cell cycle, apoptosis, signaling pathway, invasion and metastasis were evaluated, relative to DKK1 levels. In conclusion, the results of the present study indicated that DKK1 functioned as a key regulator in the progression of NSCLC. The results confirmed the differential expression of DKK1 in NSCLC cells, which may present a potential therapeutic target for cancer prevention.
\end{abstract}

Correspondence to: Dr Xiao Jun Xiang, Department of Oncology, The First Affiliated Hospital of Nanchang University, 17 Yongwaizheng Road, Nanchang, Jiangxi 330006, P.R. China E-mail: xiangxj1979@gmail.com

Key words: Dickkopf-1, non-small cell lung cancer, cancer prevention, differential expression

\section{Introduction}

Dickkopf-1 (DKK1) is a negative regulator of the Wnt/ $\beta$-catenin signaling pathway, which has a significant role in a variety of cellular processes, including differentiation, proliferation, cell motility and apoptosis (1). Increased DKK1 expression levels have been identified in patients with Wilms' tumors, hepatoblastoma, multiple myeloma and breast cancer, suggesting a potential role for DKK1 in carcinogenesis $(2,3)$. Studies have reported that the expression and roles of DKK1 differ between types of cancer, and that increased expression of DKK1 is common amongst numerous malignant tumors, including breast and lung cancer, as well as esophageal carcinomas $(4,5)$, supporting the hypothesis for a potential oncogenic function of DKK1 (6).

Inhibition of the Wnt pathway by secreted DKK1 has been shown to initiate carciogenesis in vertebrate embryos (7), and the overexpression of DKK1 has been described in multiple myeloma, hepatoblastoma and Wilms' tumor, as well as prostate, kidney, breast, lung (3) and esophageal cancers (4). The classification of types of lung cancer is based on multiple clinicopathological features (8). However, such clinical information may be incomplete or misleading in the determination of patient prognosis (9).

Increasing evidence indicates that non-small cell lung cancer (NSCLC) is one of the most common malignancies in China (10-12). Studies regarding patients with NSCLC have reported the potential predictive implication of biological and molecular parameters, including Kirsten rat sarcoma viral oncogene homolog mutations (13), c-erbB2 overexpression (14) and p53 mutations (15). Despite significant advances in cancer treatment, the enduring survival of NSCLC cells has remained elusive. To the best of our knowledge, few studies have been published investigating the consequences of DKK1 expression in NSCLC. The present study therefore aimed to reveal the expression pattern of DKK1 and its role as a carcinogenic factor in human NSCLC cell lines and tissues.

\section{Materials and methods}

Patient samples. NSCLC tissue samples were collected from 123 patients from The First Affiliated Hospital of Nanchang University (Nanchang, China) in 2010 and 2013, and formalin fixed (Sigma-Aldrich, St. Louis, MO, USA) for 
immunohistochemical staining. Age-matched normal tissue samples from 18 patients with primary NSCLC were used as a control. Tissue samples were stored and ground in liquid nitrogen to isolate total RNA and protein. Written informed consent was provided by all patients who participated in the study. The study was approved by the ethics committee of The First Affiliated Hospital of Nanchang University (Nanchang, China) and protocols were performed according to their ethical guidelines.

Cell culture. The lung squamous cell line YTMLC-9, carcinoma cell lines A549, SPC-A-1, LTEP-a-2, GLC82, A2 and PC-9 as well as large-cell lung carcinoma cell lines NCI-H460, 95C and 95D were purchased from the American Type Culture Collection (Manassas, VA, USA). Cells were cultured in RPMI-1640 medium (Invitrogen Life Technologies, Carlsbad, CA, USA), supplemented with $10 \%$ fetal bovine serum (Gibco-BRL, Invitrogen Life Technologies), 100 IU/ml penicillin and $100 \mathrm{mg} / \mathrm{ml}$ streptomycin (Life Technologies, Carlsbad, CA, USA), maintained at $37^{\circ} \mathrm{C}$ in $5 \% \mathrm{CO}_{2}$ atmospheric air. The cells were cultured to $80 \%$ confluence prior to transfection with recombined eukaryotic or empty vectors using Lipofectamine ${ }^{\circledR} 2000$ (Invitrogen Life Technologies) according to the manufacturer's instructions. For the transfection of cells, the pZERO-mcs mammalian expression vector was used (Invitrogen Life Technologies). This vector contains a hybrid EF1a/HTLV promoter, allowing efficient transcription of the recombinant DKK1-cDNA, and the puromycin resistance gene for easy selection of the transfected cells. The full-length sequence of DKK1 cDNA was isolated from the recombinant TA plasmid used for subcloning (Invitrogen Life Technologies) with the following primers: Forward, 5'-CAA GGGGATCCCCCTGCAGTCAGGACTCTGGGAC-3' and reverse, 5-GTGTTCTGCTAGCTAGGTATTATTAATTTA TTGGAAAC-3 (including Bam HI and NheI sites). The amplified DKK1 full-length cDNA was subsequently subcloned into the pZERO-mcs expression vector. The PCR product was gel-purified and digested with BamHI and NheI, then ligated into the plasmid pZERO-mcs, resulting in the recombinant plasmid pZERO-mcs-Dkk1. Transfection of the cells with either the DKK1-cDNA or an empty vector was performed using Lipofectamine 2000 (Invitrogen Life Technologies), according to the manufacturer's instructions.

Western blot analysis. The protein expression levels of DKK1 were evaluated by western blot analysis. Proteins extracted from NSCLC specimens and cell lines were used for this analysis. Tissue samples and cells were lysed in radioimmunoprecipitation buffer (Cell Signaling Technology, Danvers, MA, USA) at $4^{\circ} \mathrm{C}$ for $30 \mathrm{~min}$. The lysate was incubated on ice for $20 \mathrm{~min}$, followed by sonication for $30 \mathrm{sec}$, after which the lysate was incubated on ice for a further $15 \mathrm{~min}$. The lysate was then centrifuged at $10,000 \mathrm{x}$ g for $10 \mathrm{~min}$ in a microcentrifuge tube, and the supernatant was maintained at $-80^{\circ} \mathrm{C}$. The protein concentration was quantified using the Bradford method (16). The soluble proteins $(10-20 \mu \mathrm{g})$ were separated by 10\% SDS-PAGE and blotted onto polyvinylidene fluoride membranes (Sigma-Aldrich). Subsequently, the membranes were blocked with $5 \%$ nonfat dry milk in $1 \mathrm{X}$ Tris-buffered saline and Tween 20 buffer (TBST) at room temperature for $1 \mathrm{~h}$, prior to incubation with rabbit anti-human DKK1 polyclonal antibody (1:1,000; cat. no. LS-A2867; LifeSpan Biosciences, Inc., Seattle, WA, USA) and rabbit polyclonal anti- $\beta$-actin (1:1,000; cat. no. A2066; Sigma-Aldrich) at $25^{\circ} \mathrm{C}$ for $90 \mathrm{~min}$. Following rinsing with TBST containing $3 \%$ bovine serum albmin (BSA; Bio-Rad Laboratories, Inc., Hercules, CA, USA), the membranes were incubated with secondary antibody (Santa Cruz Biotechnology, Dallas, TX, USA) for $1 \mathrm{~h}$ at room temperature and the immunostained bands were subsequently visualized using enhanced chemiluminescence (Pierce, Thermo Fisher Scientific, Rockford, IL, USA). The intensity of each band was normalized to $\beta$-actin, the internal control, and the relative intensities were analyzed with ImageJ 1.0 software (National Institutes of Health, Bethesda, MD, USA).

Reverse transcription-quantitative polymerase chain reaction (RT-qPCR). Total RNA was extracted from the NSCLC cells using Quick-RNA ${ }^{\mathrm{TM}}$ MicroPrep solution (Zymo Research Corp., Orange, CA, USA) according to the manufacturer's instructions. Subsequently, the purified total RNA was reverse transcribed with the iScript ${ }^{\mathrm{TM}}$ Reverse Transcription supermix for PCR (Bio-Rad Laboratories, Inc.) according to the manufacturer's instructions. The reverse transcribed RNA was subjected to PCR using the SsoFast ${ }^{\mathrm{TM}}$ EvaGreen $^{\circledR}$ supermix (Bio-Rad Laboratories, Inc.). Primer sequences were designed using the OligoPerfect ${ }^{\mathrm{TM}}$ Designer 1.0 software (Invitrogen Life Technologies), according to the manufacturer's instructions for optimal primer design, and were synthesized commercially (Generay Biotech Co., Ltd., Shanghai, China). The primer sequences used in the present study are listed in Table I. The PCR cycling conditions were as follows: Initial densturation at $35^{\circ} \mathrm{C}$ for $30 \mathrm{sec}$, followed by 30 cycles at $95^{\circ} \mathrm{C}$ for $30 \mathrm{sec}, 55^{\circ} \mathrm{C}$ for $60 \mathrm{sec}$ and $68^{\circ} \mathrm{C}$ for $2 \mathrm{~min}$, and final extension at $68^{\circ} \mathrm{C}$ for $10 \mathrm{~min}$. Each reaction was performed in triplicate and three independent experiments were conducted. A standard curve was constructed using serial dilutions of a reference sample and was included in each run to correct potential variations in amplification efficiency. The relative copy numbers were obtained from the standard curve and normalized to the values for $\beta$-actin. The fold-change in expression was calculated using the $2^{-\triangle \Delta C T}$ method.

Histopathological analysis. Histopathological analyses were performed using immunohistochemical and immunofluorescent staining methods. The NSCLC samples collected from patients had previously been embedded in paraffin blocks. The paraffin-embedded sections were deparaffinized with xylene (Sigma-Aldrich) and rehydrated in graded ethanol solutions. Endogenous peroxidase activity was blocked by incubation with $3 \% \mathrm{H}_{2} \mathrm{O}_{2}$ (Sigma-Aldrich) for $15 \mathrm{~min}$ at room temperature. Sections were subsequently heated with $0.01 \mathrm{M}$ citrate ( $\mathrm{pH} 6.0$; Sigma-Aldrich) at $95^{\circ} \mathrm{C}$ for $15 \mathrm{~min}$ in a microwave (220 watts) for antigen retrieval. Following incubation with rabbit polyclonal anti-DKK1 antibody (Abcam Inc., Cambridge, MA, USA) for $2 \mathrm{~h}$ at room temperature the sections were incubated with horseradish peroxidase-labeled anti-rabbit immunoglobulin G secondary antibody. The primary antibody was omitted in the negative controls. The intensity of DKK1 staining was evaluated in tumor cells in the cytoplasm, thereby determining the immunoreactivity of DKK1 in the tumor cells. 
Table I. Primer sequences used for polymerase chain reaction analysis.

\begin{tabular}{|c|c|c|}
\hline Gene name & Primer sequence $\left(5^{\prime}-3^{\prime}\right)$ & Product size (bp) \\
\hline DKK1 & $\begin{array}{l}\text { CAACGCTATCAAGAACCTGC } \\
\text { GATCTTGGACCAGAAGTGTC }\end{array}$ & 168 \\
\hline$\beta$-actin & $\begin{array}{l}\text { CCAACCGCGAGAAGATGA } \\
\text { CCAGAGGCGTACAGGGATAG }\end{array}$ & 200 \\
\hline Cyclin D1 & $\begin{array}{l}\text { ACGAAGGTCTGCGCGTGTT } \\
\text { CCGCTGGCCATGAACTACCT }\end{array}$ & 323 \\
\hline $\mathrm{Bcl}-2$ & $\begin{array}{l}\text { TCCGCATCAGGAAGGCTAGA } \\
\text { AGGACCAGGCCTCCAAGCT }\end{array}$ & 113 \\
\hline BAX & $\begin{array}{l}\text { GGGTGGTTGGGTGAGACTC } \\
\text { AGACACGTAAGGAAAACGCATTA }\end{array}$ & 199 \\
\hline Akt-1 & $\begin{array}{l}\text { GCACAAACGAGGGGAGTACAT } \\
\text { CCTCACGTTGGTCCACATC }\end{array}$ & 113 \\
\hline MMP2 & $\begin{array}{l}\text { GGCCCTGTCACTCCTGAGAT } \\
\text { GGCATCCAGGTTATCGGGGA }\end{array}$ & 474 \\
\hline VEGFC & $\begin{array}{l}\text { AGGAGGGCAGAATCATCACG } \\
\text { TATGTGCTGGCCTFGGTGAG }\end{array}$ & 405 \\
\hline
\end{tabular}

$\mathbf{A}$

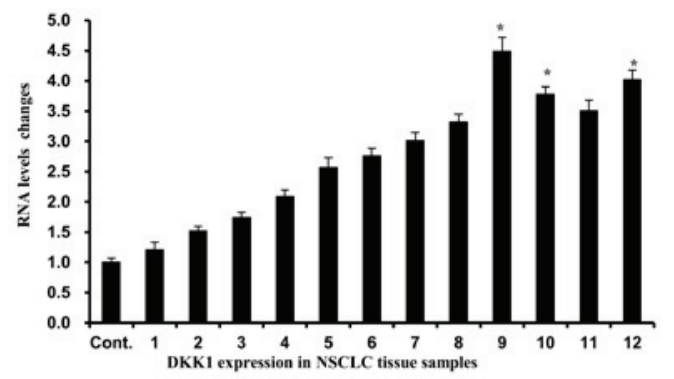

B

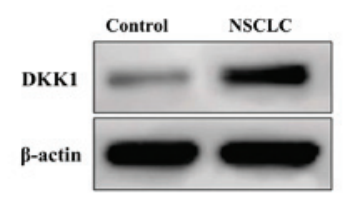

C
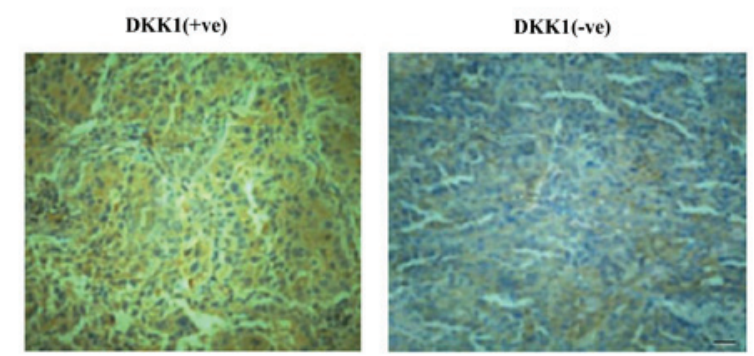

Figure 1. Differential expression of DKK1 in human NSCLC tissues. (A) Messenger RNA levels of DKK1 in various NSCLC tissue samples. Values are expressed as the mean \pm standard deviation. (B) DKK1 protein expression in representative normal and cancerous (NSCLC) tissues. $\beta$-actin was used as an internal control in the polymerase chain reaction blots. (C) Immunohistochemical staining pattern of representative DKK1-positive and -negative tissues (magnification, x100). DKK1, Dickkopf-1; NSCLC, non-small cell lung cancer.

TE13 cells were cultured on glass coverslips, fixed with $4 \%$ paraformaldehyde and permeabilized with $0.2 \%$ Triton X-100 in phosphate-buffered saline (PBS) for $10 \mathrm{~min}$ at room temperature. Cells were subsequently blocked with $3 \%$ BSAfor 30 min at room temperature, and incubated with primary antibodies diluted in PBS supplemented with $3 \%$ BSA for 60 min at room temperature. Following washing with PBS, the cells were stained with fluorescein isothiocyanate-conjugated secondary antibody (Santa Cruz Biotechnology, Inc.) for $30 \mathrm{~min}$ at $37^{\circ} \mathrm{C}$. Finally, the coverslips were washed with PBS and the nuclei were stained with DAPI (Sigma-Aldrich) or rhodamine B (Sigma-Aldrich) and visualized with a confocal laser scanning microscope (Leica SP5; Leica Microsystems, Wetzlar, Germany). The images were analyzed using Leica LAS AF software (Leica Microsystems).

Statistical analysis. Statistical analyses were performed using SPSS version 14.0 (SPSS Inc., Chicago, IL, USA) software. Values are presented as the mean \pm standard deviation. Student's t-test, one-way analysis of variance and $\chi^{2}$ test were performed. $\mathrm{P}<0.05$ was considered to indicate a statistically significant difference. All experiments were performed at least in triplicate.

\section{Results}

DKK1 is expressed in NSCLC tissues. DKK1 expression levels in the NSCLC samples collected from 123 patients were evaluated and compared with those of age-matched normal tissue from patients with low-grade NSCLC. Evaluation included quantification of DKK1 gene and protein expression levels and immunohistochemical analyses. For the analysis of protein and gene expression, 25 samples of cancerous tissue and 15 samples of age-matched normal tissue were evaluated (17). The mRNA expression levels of DKK1 in cancer tissues were found to be two- to six-fold greater than those in normal tissues (Fig. 1A). Similarly, DKK1 protein expression levels were found to be 


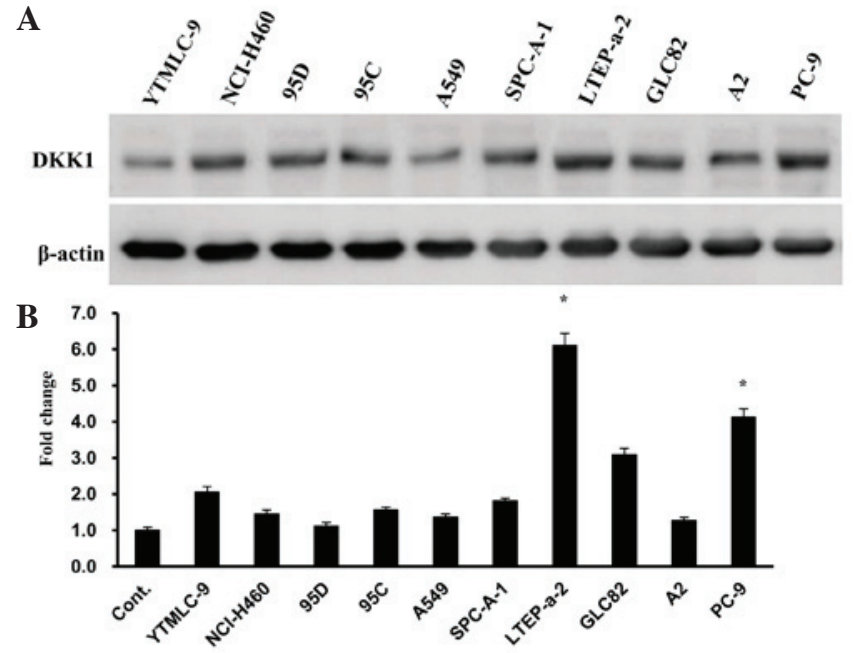

C
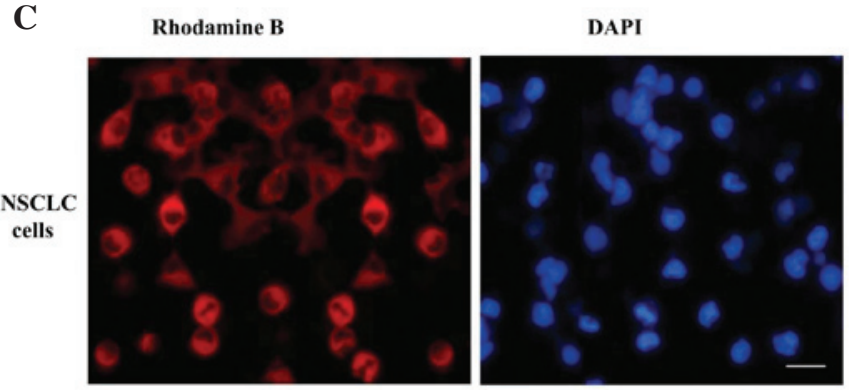

Figure 2. Differential expression of DKK1 in various NSCLC cell lines (A) DKK1 protein expression levels in ten NSCLC cell lines. (B) DKK1 messenger RNA expression levels in ten NSCLC cell lines. Values are expressed as the mean \pm standard deviation. (C) Immunohistochemical staining of DKK1 in NSCLC cells. DKK1 protein appeared as fine-grained particles in the cytoplasm, visualized with rhodamine B staining (red). DAPI staining (blue) indicates the cell nuclei (magnification, $\mathrm{x} 100$ ). ${ }^{*} \mathrm{P}<0.05$, compared with control. DKK1, Dickkopf-1; NSCLC, non-small cell lung cancer.

significantly greater in cancer tissue samples than those of the normal tissue samples (Fig. 1B). These results suggested a potential role for DKK1 in cancer progression. Clinical samples were obtained from 98 patients were subjected to immunohistochemical analysis. Of the 98 samples analyzed, 62 (63.3\%) were positively stained for DKK1 and 36 (37\%) demonstrated negative staining (Fig. 1C). Furthermore, no immunohistopathological staining for DKK1 was observed in normal tissues. These results indicated that increased expression of DKK1 is common in human NSCLC, which may potentially be associated with the metastasis of NSCLC.

DKK1 is differentially expressed amongst various NSCLC cell lines. DKK1 expression was evaluated in ten human NSCLC cell lines. Western blot analysis revealed that DKK1 protein was detected in all ten cell lines, but that the expression levels varied amongst the cell lines. A significantly increased level of DKK1 protein was observed in the LTEP-a-2, GLC-82 and PC-9 cell lines. The lowest levels of DKK1 protein were observed in the A2 and 95C cell lines (Fig. 2A). The mRNA expression levels of DKK1 were quantified using RT-qPCR, and the results were concurrent with those of the protein expression levels of corresponding cell lines (Fig. 2B). The LTEP-a-2 cell line demonstrated significantly (six-fold) increased mRNA expression levels. However, the minimum mRNA expression levels of
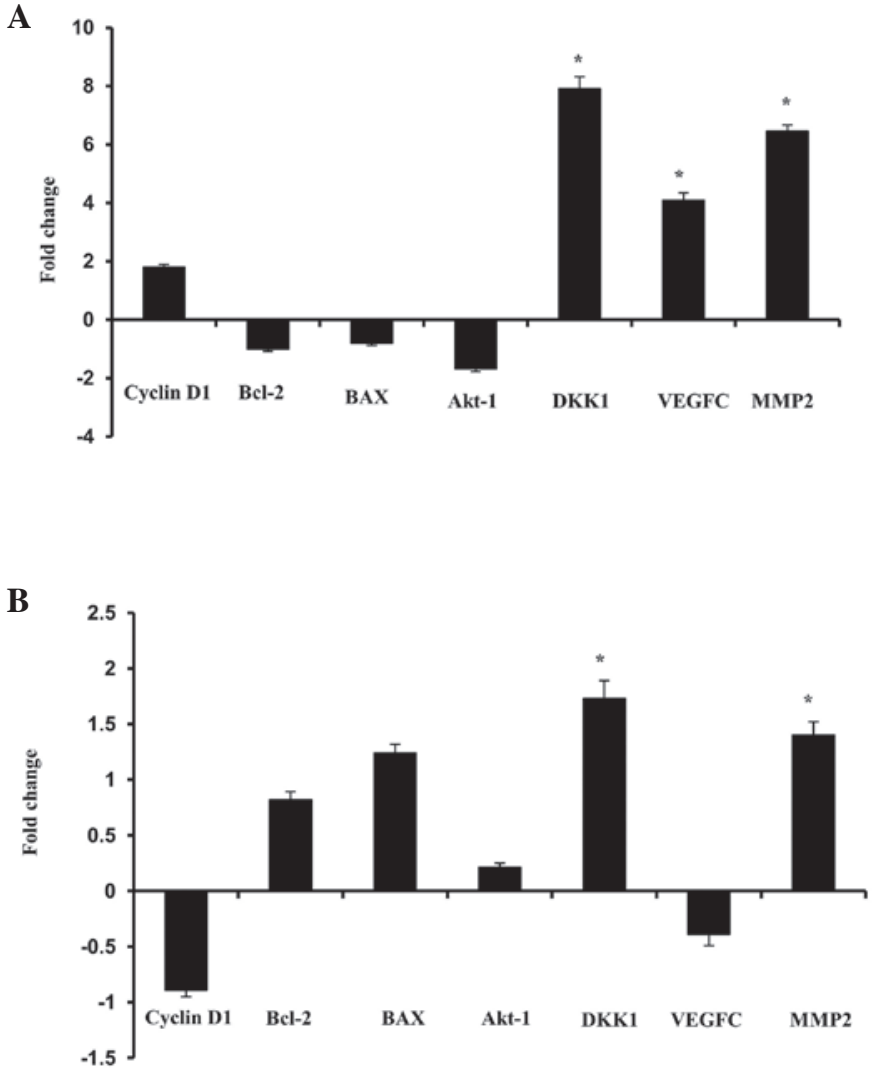

Figure 3. Differential gene expression pattern of key genes involved in the cell cycle, apoptosis, signaling pathways, invasion and metastasis with respect to DKK1 expression. Gene expression profile of (A) LYEP-a-2 cells and (B) 95D cells. * $\mathrm{P}<0.05$, compared with control.

DKK1 were observed in 95D cells, compared with those of the other cell lines. Immunofluorescent staining with rhodamine B revealed the sub-cellular localization of DKK1 protein, which was determined by the detection of rhodamine B-stained, fine-grained particles in the cytoplasm (Fig. 2C). The corresponding cell nuclei were stained with DAPI.

Regulatory effect of DKK1 in NSCLC cell lines. The involvement of DKK1 in the cell cycle (cyclin D1), apoptosis (Bcl-2, BAX), signaling pathways (Akt-1), as well as invasion and metastasis (MMP2, VEGC) in selected cell lines with high (LTEP-a-2) and low (95D) mRNA expression of DKK1, were evaluated. A relative differential gene expression profile with respect to the expression of DKK1 was constructed for the two cell lines (Fig. 3). The gene expression profile in LYEP-a-2 cells revealed increased mRNA levels of cyclin D1, MMP2 and VEGC. However, the levels of Bcl-2, BAX and Akt-1 were found to be significantly decreased (Fig. 3A). By contrast, the gene expression profile in $95 \mathrm{D}$ cells revealed a contrary expression with respect to the low levels of DKK1 expression. The mRNA expression levels of cyclin D1, MMP2 and VEGC were decreased concurrently with the decreased levels of DKK1. However, the levels of Bcl-2, BAX and Akt1 were found to be increased (Fig. 3B). These results demonstrated a regulatory effect of the DKK1 gene in the suppression of cell cycle, signaling pathways and apoptosis in NSCLC cells. In addition, enhanced DKK1 gene expression facilitates the cell cycle, invasion and metastasis (6). 


\section{Discussion}

The secreted protein DKK1, an antagonist of the Wnt/ $\beta$-catenin signaling pathway, has been implicated in tumor progression (4) and found to be expressed in multiple types of human cancer (4). The role of DKK1 in tumor progression may differ depending on the cell type (5). DKK1 is upregulated in certain types of human cancer, including NSCLC, hepatocellular carcinoma and pancreatic cancer $(5,18)$.

Emerging studies have focused on elucidating the significance of DKK1 in mediating tumor progression; however, the biological effects of DKK1 in NSCLC cells have remained elusive. The present study aimed to identify the differential expression of DKK1 in NSCCLC cells, including those obtained from human cancerous tissue specimens and in certain NSCLC cell lines. The present study also aimed to confirm the involvement of DKK1 in the regulation of tumor progression via the modulation of key genes involved in the cell cycle, apoptosis, cell invasion and metastasis. DKK1 expression was therefore evaluated in human NSCLC tissue specimens and ten NSCLC cell lines. DKK1 protein was expressed in almost all the NSCLC cancer cells evaluated; however, the expression levels varied between tissues and cell lines. DKK1 protein and gene were expressed in analogous patterns, with respect to the type of tissue or cell line. Immunohistochemical analysis of clinical samples from 98 NSCLC patients revealed DKK1-positive staining in $63.3 \%$ of the samples and the remaining $37 \%$ with negative staining. Furthermore, no staining for DKK1 was detected in non-cancerous or normal cells. These results implicated DKK1 in the progession of NSCLC, which was consistent with the results of previous studies $(4,5,19)$.

Studies previously hypothesized that DKK1 was involved in the downstream targeting of $\beta$-catenin/T-cell factor and participated in a negative feedback loop within the Wnt signaling pathway of colon cancer cells $(1,20)$. In addition, studies revealed that the overexpression of DKK1 was associated with poor patient prognosis $(5,21,22)$, whereas no clear evidence regarding the function of DKK1 in NSCLC was identified.

The present study aimed to elucidate the molecular mechanisms underlying the effects of DKK1 in tumor progression. Two NSCLC cell lines, which demonstrated significantly increased (LYEP-a-2 cells) and decreased (95D cells) levels of DKK1 mRNA expression were selected. The relative expression levels of key proteins involved in the cell cycle (cyclin D1), apoptosis (Bcl-2, BAX), signaling pathway (Akt-1), invasion and metastasis (MMP2, VEGC) were examined with respect to DKK1 expression levels. Of note, a proportional increase in genes associated with the cell cycle, as well as invasion and metastasis, were detected concurrently with high levels of DKK1 gene expression in LYEP-a-2 cells. Furthermore, the high levels of DKK1 in LYEP-a-2 cells were associated with decreased expression levels of genes involved in apoptosis and signaling pathways. These results were supported by those obtained from the analysis of gene expression in 95D cells, where low DKK1 expression levels were associated with inverse alterations in gene expression. Low expression levels of DKK1 induced increases in the mRNA expression levels of apoptotic genes Bcl-2 and BAX, as well as signaling pathway-associated Akt-1. The results therefore indicated that increased DKK1 gene expression may enhance the cell cycle, as well as invasion and metastasis of NSCLC cells. However, further studies are required to elucidate the specific mechanisms underlying this biological effect.

Conversely, previous studies have demonstrated that DKK1 suppressed cell growth and migration $(6,23)$, suggesting that DKK1 may have diverse biological roles in distinct types of cancer cell. To date, few studies have been published regarding the role of DKK1 in NSCLC, and these have mainly focused on elucidating its diagnostic or prognostic value (24-26). Further studies are required to elucidate the mechanisms underlying the differential and relative expression of DKK1 in NSCLC.

In conclusion, the present study confirmed the involvement of DKK1 in NSCLC progression. The results revealed differential expression of DKK1 in NSCLC cells, which may provide a potential therapeutic target for cancer prevention, particularly in NSCLC.

\section{References}

1. Niida A, Hiroko T, Kasai M, et al: DKK1, a negative regulator of Wnt signaling, is a target of the beta-catenin/TCF pathway. Oncogene 23: 8520-8526, 2004.

2. Gavriatopoulou M, Dimopoulos MA, Christoulas D, et al: Dickkopf-1: a suitable target for the management of myeloma bone disease. Expert Opin Ther Targets 13: 839-848, 2009.

3. Forget MA, Turcotte S, Beauseigle D, et al: The Wnt pathway regulator DKK1 is preferentially expressed in hormone-resistant breast tumours and in some common cancer types. Br J Cancer 96: 646-653, 2007.

4. Darlavoix T, Seelentag W, Yan P, Bachmann A and Bosman FT: Altered expression of CD44 and DKK1 in the progression of Barrett's esophagus to esophageal adenocarcinoma. Virchows Arch 454: 629-637, 2009.

5. Makino T, Yamasaki M, Takemasa I, et al: Dickkopf-1 expression as a marker for predicting clinical outcome in esophageal squamous cell carcinoma. Ann Surg Oncol 16: 2058-2064, 2009.

6. Wirths O, Waha A, Weggen S, Schirmacher P, Kühne T, et al: Overexpression of human Dickkopf-1, an antagonist of wingless/WNT signaling, in human hepatoblastomas and Wilms' tumors. Lab Invest 83: 429-434, 2003.

7. Marvin MJ, Di Rocco G, Gardiner A, et al: Inhibition of Wnt activity induces heart formation from posterior mesoderm. Genes Dev 15: 316-327, 2001.

8. Mountain CF: Revisions in the international system for staging lung cancer. Chest 111: 1710-1717, 1997.

9. Martini N, Bains MS, Burt ME, et al: Incidence of local recurrence and second primary tumors in resected stage I lung cancer. J Thorac Cardiovasc Surg 109: 120-129, 1995.

10. Chen WQ, Zheng RS and Zeng HM: Bayesian age-period-cohort prediction of lung cancer incidence in China. Thoracic Cancer 2: 149-155, 2011.

11. Chen W, Zheng R, Zhang S, Zou X, Zhao P, et al: Lung cancer incidence and mortality in China, 2009. Thoracic Cancer 4: 102-108, 2013.

12. Zeng H, Zheng R, Zhang S, He J and Chen W: Lung cancer incidence and mortality in China, 2008. Thoracic Cancer 4: 53-58, 2013.

13. Fukuyama Y, Mitsudomi T, Sugio K, et al: K-ras and p53 mutations are an independent unfavourable prognostic indicator in patients with non-small-cell lung cancer. Br J Cancer 75: 1125-1130, 1997.

14. Osaki T, Mitsudomi T, Oyama T, Nakanishi R and Yasumoto K: Serum level and tissue expression of c-erbB-2 protein in lung adenocarcinoma. Chest 108: 157-162, 1995.

15. Oyama T, Osaki T, Mitsudomi T, et al: p53 alteration, proliferating cell nuclear antigen and nucleolar organizer regions in thymic epithelial tumors. Int J Mol Med 1: 823-826, 1998.

16. Bradford MM: A rapid and sensitive method for the quantitation of microgram quantities of protein utilizing the principle of protein-dye binding. Anal Biochem 72: 248-254, 1976. 
17. Tammemägi MC, Katki HA, Hocking WG, et al: Selection criteria for lung-cancer screening. New Engl J Med 368: 728-736, 2013

18. Takahashi N, Fukushima T, Yorita K, Tanaka H, Chijiiwa K, et al: Dickkopf- 1 is overexpressed in human pancreatic ductal adenocarcinoma cells and is involved in invasive growth. Int $\mathrm{J}$ Cancer 126: 1611-1620, 2010.

19. Yamabuki T, Takano A, Hayama S, Ishikawa N, Kato T, et al: Dikkopf-1 as a novel serologic and prognostic biomarker for lung and esophageal carcinomas. Cancer Res 67: 2517-2525, 2007

20. González-Sancho JM, Aguilera O, García JM, et al: The Wnt antagonist DICKKOPF-1 gene is a downstream target of beta-catenin/TCF and is downregulated in human colon cancer. Oncogene 24: 1098-1103, 2005.

21. Yu B, Yang X, Xu Y, Yao G, Shu H, et al: Elevated expression of DKK1 is associated with cytoplasmic/nuclear beta-catenin accumulation and poor prognosis in hepatocellular carcinomas. J Hepatol 50: 948-957, 2009.
22. Sheng SL, Huang G, Yu B and Qin WX: Clinical significance and prognostic value of serum Dickkopf-1 concentrations in patients with lung cancer. Clin Chem 55: 1656-1664, 2009.

23. Qin X, Zhang H, Zhou X, Wang C, Zhang X, et al: Proliferation and migration mediated by Dkk-1/Wnt/beta-catenin cascade in a model of hepatocellular carcinoma cells. Transl Res 150: 281-294, 2007.

24. Yao X, Jiang H, Zhang C, Wang H, Yang L, et al: Dickkopf-1 autoantibody is a novel serological biomarker for non-small cell lung cancer. Biomarkers 15: 128-134, 2010.

25. Osada H, Tomida S, Yatabe Y, Tatematsu Y, Takeuchi T, et al: Roles of achaete-scute homologue 1 in DKK1 and E-cadherin repression and neuroendocrine differentiation in lung cancer. Cancer Res 68: 1647-1655, 2008.

26. Licchesi JD, Westra WH, Hooker CM, Machida EO, Baylin SB, et al: Epigenetic alteration of Wnt pathway antagonists in progressive glandular neoplasia of the lung. Carcinogenesis 29: 895-904, 2008 